\title{
Colloid Transport and Retention in Fractured Deposits
}

\author{
Oak Ridge National Laboratory \\ September 1997 \\ Progess Report
}

Principal Investigator

John F. McCarthy

(423) 576-6606 (Phone)

(423) 576-3989 (Fax)

jjj@ornl.gov

Oak Ridge National Laboratory

P.O. Box 2008, Bethel Valley Road

Oak Ridge, Tennessee 37831-6036

\section{Co-Investigators}

Larry D. McKay

(423) 974-0821 (Phone)

(423) 974-2368 (Fax)

mckay@yoda.gg.utk.edu

university of Tennessee

Knoxville, TN 37931

Motomu Ibaraki

(614) 292-7528 (Phone)

(614) 292-7688 (Fax)

ibaraki@geology.ohio-state.edu

Ohio State University

Columbus, OH 43210-1308
Paul Reimus

(505) 665-2537 (Phone)

(505) 667-8487 (Fax)

preimus@lanl.gov

Los Alamos National Laboratory

Los Alamos, NM 87545

Martha J. M. Wells

(615) 372-6123 (Phone)

mjw5030@tntech.edu

Tennessee Technological University

Cookeville, TN 38501 


\section{Research Objective}

The goal of this project is to identify the chemical and physical factors that control the transport of groundwater colloids in fractured porous media and develop a generalized capability to predict colloid attachment and detachment based on hydraulic factors (head, flow rate), physical structure (fracture aperture), and chemical properties (surface properties of colloids and fracture surfaces). Understanding the processes that control colloid behavior will increase the confidence with which colloid-facilitated contaminant transport can be predicted and assessed at various contaminated U.S. Department of Energy (DOE) sites. An added benefit is the expectation that this work will yield novel techniques to either immobilize colloid-bound contaminants in situ or mobilize colloids for enhancing remedial techniques such as pump-andtreat and bioremediation.

\section{Research Statement}

A series of field-scale and laboratory-scale experiments, using both natural undisturbed samples and simple one-dimension "artificial fractures," are in progress to investigate the influence of physical and chemical factors on the transport of colloids in fractured materials. The experimental results will be assessed using a computer model (COLFRAC) developed to simulate colloid transport in fractured materials. The overall goal is to assess the relative influence of chemical and physical factors expected to influence colloid transport in fractured materials and investigate strategies for predictive simulation at the field scale. The experimental methods each operate at different physical/geological scales and can be used with different degrees of experimental control. This allows testing of hypotheses in a relatively simple setting in the laboratory where individual chemical or colloidal characteristics can be varied and then the results compared with field-scale experiments where the influence of realistic geologic heterogeneity can be incorporated.

The work is organized into interacting tasks dealing with theoretical descriptions of colloid transport in fractures, transport studies at three spatial scales (simple one-dimensional fractures, laboratory columns of intact geological material, and field-scale colloid tracer studies), and computer modeling of colloid transport processes. A continuing iteration among all tasks and experimental scales is envisioned throughout the project.

Predictions based on laboratory experiments in simplified artificial fracture systems will be tested in column and field studies, and, likewise, hypothesized interpretations of the results of the column or field studies will be tested and verified in additional laboratory studies. Experimental efforts at all scales will begin with simple binary comparisons (e.g., large vs small colloids with similar surface chemistry), and proceed with increasing complexity (e.g., varying surface properties of colloids or fractures) as understanding is developed. It is only through this parallel iteration at different scales that predictions based on laboratory understanding can be tested in column and in field studies so that additional research-can be conducted to resolve observations that were not consistent with the earlier descriptions of controlling processes. 


\section{Research Progress}

In FY 1997, progress was made in all phases of research. Some research components benefitted from preliminary work in progress before- the funding of the EMSP project, and this is reflected in the lists of publications and abstracts.

\section{Theoretical Descriptions of Colloid Transport in Fractures}

Theoretical relationships used to describe colloid transport in porous media (filtration theory) are being reformulated to account for the physics of settling, diffusion, and collision of colloids with fracture walls rather than individual grains of porous media. An experimental system has been set up and tested to evaluate and refine theoretical predictions. This simple, onedimensional "artificial fracture" can be varied with respect to fluid flow rate and aperture thickness. Chemical interactions between colloids and fractures will be varied by modifying the aqueous chemistry and the material used as the walls of the artificial fracture.

Ideally for these theoretical studies, the surfaces of the "fracture walls" need to be chemically uniform and very smooth, with surface roughness smaller than the dimensions of the colloids transported in the fracture. Collaboration with material scientists at ORNL has identified sol-gel processes used in the production of silicon wafers that will be used to fabricate "fracture walls" of silica oxide and titanium dioxide, both of which have well-characterized surface charge properties. Initial experiments have used negatively charged polysulfonate membranes as fracture walls and demonstrated that the breakthrough of fluorescence-tagged microspheres can be quantified using a chromatographic fluorescence detector.

\section{Intact Column Studies}

Experimental undisturbed column samples of weathered fractured saprolite and unweathered fractured shale have been used to investigate the effects of physical and chemical factors on colloid transport. Column studies incorporate the natural heterogeneity in fracture dimensions and surface properties that cannot be simulated in the artificial fracture studies, but can be more readily characterized than larger field-scale systems. During the first year of the project, we completed evaluation of a series of laboratory experiments on the influence of particle diameter on colloid transport in undisturbed columns of fractured shale saprolite.

The experiments showed that there was an optimum size of 0.5 to $1.0 \mu \mathrm{m}$ for transport of the latex microsphere tracers. Simple one-dimensional simulations suggest that gravitational settling was the principal mechanism for removal of the larger-than-optimum particles. Loss of the smaller-than-optimum particles was attributed mainly to their more frequent collisions with the fracture walls, which is strongly influenced by diffusion in the fractures. Dismantling the soil column and mapping the distribution of the microspheres clearly showed evidence of the size segregation of particles within the fractures, often resulting in clearly defined "channels" which contained all sizes of the microsphere tracers. Measured profiles in the sample showed that the smaller microspheres $(<0.1 \mu \mathrm{m})$ were also able to penetrate up to $4 \mathrm{~mm}$ into the fine-grained matrix between fractures.

This study and a previous study on the influence of flow rate clearly show that the physical factors of particle size and flow rate play a major role in controlling transport and retention of particles in fractured saprolite. Generally, the behavior of colloidal tracers in the fractured 
saprolite is consistent with colloid filtration theory developed for granular materials. However, because of the wider range of flow rates typically observed in fractured materials, flow velocity appears to play a greater role.

A series of experiments to examine the influence of geochemical factors on colloid transport is currently under way. The initial set of experiments involves carrying out repeated tracer experiments using fluorescent microspheres in carrier solutions with a range of values of ionic strength and monovalent/divalent cation ratios. At low ionic strengths (such as might occur during rapid infiltration of precipitation) or in solutions dominated by monovalent cations, electrostatic attraction to the clay minerals should be minimal. As ionic strength is increased, greater losses are expected. By carrying out experiments with both varying chemistry and varying particle size, we expect to be able separate the relative influence of the physical and electrostatic factors. This data will then be used for comparisons with field experiments and for testing of colloid transport models.

\section{Field Tracer Experiments}

The effect of colloid properties on their transport was measured in a fractured highlyweathered shale saprolite. Field studies are essential to identify processes that emerge as important only in complex natural systems and to verify hypotheses that attempt to integrate multiple interactive processes in natural systems. This year's field experiment focused on the influence of particle diameter on colloid transport in the field, and results were compared to those from the intact column studies (above). The objective was to determine whether colloid transport at the field scale is affected by the spatial distribution of physical and chemical features along a flow path and by kinetic processes at spatial and temporal scales that cannot be predicted or understood from column experiments. The experiment was conducted at a site in fractured, highly weathered saprolite in the same formation from which the intact column was excavated. The site was within a Solid Waste Storage Area at ORNL; since colloid-facilitated transport of transuranics has been observed at this site, the results of the field experiment are directly relevant to DOE concerns.

Three sizes of fluorescent microspheres and a nonreactive tracer (fluorobenzoic acid) were injected into each of two source wells and arrival of colloids monitored in a downgradient well. A different color of microspheres and a different flurobenzoate isomer were used in each injection well. Because each injection well had a distinctive color of microsphere and chromatographically-distinct nonreactive tracer, the extent of transport to the monitoring well along two flow paths of different lengths could be compared.

Preliminary analyses indicate that many of the patterns observed in the intact columns were observed at the field scale. As in the columns, flow velocity controls the concentration of the colloid, with higher recovery of colloids along the flow path that had a higher hydraulic gradient and faster\&rival of the nonreactive tracer. Likewise, there was an optimum size of colloid along both flow paths. Losses of very small colloids $(0.08 \mu \mathrm{m})$ were orders of magnitude greater than for the $0.8-$ and $2.0 \mu \mathrm{m}$ colloids. The field experiment also provided important information not available from column studies. Losses of colloids were not linearly proportional to distances. The greatest loss occurred over the first meter of travel, with little additional loss over the next three meters. The nonlinearity of the losses suggests that once colloids are mobilized, they may be transported over quite long distances. 
Microspheres recovered in the monitoring well were quantified by counting fluorescent particles captured after filtration of groundwater on $0.05-\mu \mathrm{m}$ filters. Not only is this procedure tedious for the large number of samples generated by a field experiment, but difficulty was encountered in counting the smallest microspheres because even the very low concentration of natural colloids present in the groundwater obscured the view of the microspheres on the filters.

Significant progress was made on the development of a flow cytometer designed to quantitatively count fluorescent colloids as small as $0.05-\mu \mathrm{m}$ diameter in groundwater samples. The design of the instrument is based on a prototype flow cytometer built by another project to detect and count fluorescentdye-tagged DNA fragments. Although the development of the cytometer is being funded primarily by other projects at Los Alamos, it will be available to analyze fluorescent colloid concentrations in groundwater samples for the EMSP project.

\section{Computer Modeling of Colloid Transport}

Computer models function not only as tools to extrapolate beyond the specific experimental results, but can help identify which processes are critical to accurate prediction and must, therefore, be well-understood. In this project, the modeling task interacts with other tasks to incorporate improved understanding of colloid transport and retention in the model. The model, COLFRAC, accounts for advective-dispersive colloid transport in the fractures, aqueous-phase contaminant transport in the fractures and the porous matrix, and sorption of the solute. The original model focused on the behavior of contaminant solutes; numerical formulations within that model allowed for either equilibrium or kinetic sorption reactions of solutes onto the fracture walls, the matrix solids, and the mobile and filtered colloids according to either a Langmuir or a Freundlich isotherm.

However, descriptions of the colloid transport process were less detailed. The code only accounted for advective-dispersive transport of colloids using a single first-order filtration coefficient to describe colloid interactions with the fractured media. Because the intact column and field studies suggested that diffusion of colloids into the fine pore structure is an important loss mechanism for colloids, an initial goal was to modify the code to include diffusion processes of colloids into the fine pore structure. The first stage of the major modification of COLFRAC to include a diffusion term is near completion. One major modification includes the incorporation of a subroutine to describe the migration of colloids traveling along a fracture into the surrounding porous matrix. This diffusion phenomenon will account for colloid migration from a main fracture into a network of smaller fractures connected to the main fracture.

Although COLFRAC can simulate colloid transport in a network of smaller fractures connected to a main fracture, this approach is computationally very expensive. Treating this phenomenon as a diffusion process would slightly increase the computational cost, and this is much more computationally efficient. The descriptions of colloid transport will also be improved by incorporating the filtration coefficients derived from the theoretical and laboratory studies described earlier. Thus, filtration coefficients can be calculated for specific fracture aperture and fluid velocity combinations within different fractures in a simulated network of variable-sized fractures. This improved model will be tested by comparing simulations of colloid transport to observed breakthroughs of different sized colloids in the intact column experiments. 


\section{Summary of Accomplishments}

- Set up and tested a one-dimensional "artificial fracture" that can be varied with respect to fluid flow rate and aperture thickness to evaluate and refine theoretical predictions.

- Completed experiments and one-dimensional simulations on the influence of particle diameter on colloid transport.

- Collected undisturbed column samples for experiments on the influence of geochemical factors on colloid transport.

- Completed colloid field transport experiment examining the influence of particle size and flow path distance on colloid transport.

- Modified COLFRAC to include a subroutine to describe the migration of colloids traveling along a fracture into the surrounding fine-porosity matrix.

- Completed and submitted one master's thesis and two journal manuscripts.

\section{Papers and Abstracts}

Cumbie, D. H. 1997. Laboratory Scale Investigations into the Influence of Particle Diameter on Colloid Transport in Highly Weathered and Fractured Shale Saprolite. MS thesis, University of Tennessee, Knoxville, Tenn., May 1997.

Cumbie, D. H. and L. D. McKay. "Influence of Particle Diameter on Colloid Transport in a Highly Weathered and Fractured Shale." Submitted to the Journal of Contaminant Hydrology. In review.

Cumbie, D. H. and L. D. McKay, "Laboratory Investigations of the Influence of Particle Diameter on Transport of Latex Microspheres in Fractured Shale Saprolite." Abstract presented at Fall Meeting of AGU, San Francisco, Calif., December 1996. (Winner of Outstanding Student Paper Award.)

Harton, A. D., L. D. McKay, and G. V. Wilson. "Influence of Flow Rate on Transport of Bacteriophage in a Highly Weathered and Fractured Shale." Submitted to Water Resources Research. In review.

McKay, L. D., D. H. Cumbie, and A. D. Harton. "Factors Influencing Colloid Transport in Fractured Shale Saprolite." Abstract accepted for presentation at Annual Meeting of GSA, Salt Lake City, Utah, October 1997. 